\title{
The Role of Residual Contaminants and Recycling Steps on Rheological Properties of Recycled Polypropylene
}

\section{Robert da Silva Paiva}

UFSCar: Universidade Federal de Sao Carlos

Isabelly Bertochi Veroneze

UFSCar: Universidade Federal de Sao Carlos

\section{Magdalena Wrona}

UNIZAR: Universidad de Zaragoza

\section{Cristina Nerín}

UNIZAR: Universidad de Zaragoza

Sandra Andrea Cruz ( $\boldsymbol{\nabla}$ sandra.cruz@ufscar.br)

Universidade Federal de Sao Carlos Centro de Ciencias Exatas e de Tecnologia https://orcid.org/00000002-5548-0166

\section{Research Article}

Keywords: polypropylene, recycling, migration, contaminants, degradation, food simulants

Posted Date: May 10th, 2021

DOI: https://doi.org/10.21203/rs.3.rs-476987/v1

License: (9) (1) This work is licensed under a Creative Commons Attribution 4.0 International License. Read Full License

Version of Record: A version of this preprint was published at Journal of Polymers and the Environment on June 22nd, 2021. See the published version at https://doi.org/10.1007/s10924-021-02214-2. 
a. Chemistry Department, Center for Exact Sciences, Federal University of São Carlos (UFSCar),

Rodovia Washington Luís, Km 235, 10 SP-310, São Carlos, Brazil. simulants.

Recycling of polymers is one of the alternatives to reduce the impact of polymers presence on the environmental. However, the contaminants, defined as non-intentionally added substances, present in recycled material may migrate into food and also change its molecular structure. This work addresses the extractability/migration of contaminants from polypropylene (PP) samples into food simulants and the influence of these contaminants on the molecular structure of recycled PP. For this PP was contaminated with several substances to simulate a "worst-case" scenario and, then it was submitted to a recycling process. Extractability tests were performed by solid-phase microextraction and gas chromatography coupled to mass spectroscopy both to evaluate the presence of contaminants in the PP samples and their ability to migrate in food simulants. Additionally, molecular changes of the PP samples were evaluated by oscillatory rheometry. After washing and extrusion of the PP samples the extractability results showed considerable reductions of migrations in the food simulants and indicated, in some cases, compliance with regulations for using recycled polymer in contact with food. The residual contaminants were present in the polymer, the high temperatures and shear rates play an important role in molecular changes. Finally, the results highlight the importance of the use of rheological measurements to detect the influence of contaminants in recycled materials. Their presence result in material with different molar mass, that may be applied in different market applications.

Keywords: polypropylene; recycling, migration; contaminants; degradation; food 
Using post-consumer-recycled polymers for food packaging has been increasing in the last few years and then, this has mitigated their environmental impact [1,2]. Despite this, the usage of direct food contact packaging has some restrictions regulated by the Food and Drugs Administration (FDA) [3], the European Regulations No. 1935/2004; 10/2011 and 282/2008 [4]; and the National Health Surveillance Agency (ANVISA) [5] in Brazil and Mercosul [6]. This concern is associated with the misuse of packaging by the consumer through contamination with, for example, pesticides, solvents and cleaning products [7-11]. The decontamination process or the use of a functional barrier must be employed after the recycling process to ensure that the final material is safe and thus the migration is negligible [12]. The efficiency of the technology employed to recycle polymers involves the use of a procedure entitled "challenge test" [13]. During the test, the pristine polymer is exposed to a cocktail of contaminants with varying physicochemical properties, volatility and polarity (volatile-nonpolar, volatile-polar, nonvolatile-polar and nonvolatile-nonpolar) and then, it goes through the recycling process. Early studies $[9,14-17]$ focus on the efficiency of the decontamination step and evaluate the migration of the surrogates to food simulants. However, most recycling technologies and studies deal with PET and few studies are dedicated to evaluating the stage of each recycling process in the decontamination of polyolefins.

However, polyolefins have lower thermal stability and products of the degradation may be formed during the processes, as described by Coulier, Orbons and Rijk [18]. Although this is an important issue, literature regarding the impact of the presence of residual contaminants on the physicochemical properties of the recycled polymer is 
scarce. Nevertheless, our research group has investigated this topic $[9,19]$. For example,

Garcia, Scuracchio and Cruz [15] evaluated the different mechanical recycling (single-

screw, co-rotating twin-screw and cascade extruder) process on the post-consumer polypropylene (PP). The molecular structure of the PP was analyzed by rheological measurements, which demonstrated that different types of processing together with contaminants showed distinct levels of degradation. In other words, the residual contaminants play an important role in the recycling process, especially for polyolefins. As described by Palkopoulus [16], most studies extrapolate the results obtained for PET to the polyolefins. Notwithstanding, two aspects need to be addressed: (i) the PP is a rubbery polymer, which means that procedure temperatures are above its glass transition temperature. Consequently, it results in a high diffusion coefficient, when compared to other glassy polymers; and (ii) the PP has reduced thermal stability, which means the presence of residual contaminants may considerably influence its structural characteristics. In this way, rheometry has long been used to provide information on physicochemical properties, principally molar mass. Cruz and coworkers [29] used of melt rheology and solution viscometry to evaluate the degradation of post-consumer poly(ethylene terephthalate). The effects of the contaminants, reprocessing and solid state polymerization were analyzed.

Therefore, this work addresses the evaluation of the migration of residual contaminants and changes in the molecular structure of recycled polypropylene. It also assesses these effects on the recycling steps, such as washing and reprocessing. Initially, to simulate the worst-case scenario of misuse, polypropylene was contaminated with a cocktail, according to an FDA protocol [20]. Then the samples were submitted to a 
performed with liquid food simulants and analysis by SPME-GC-MS to evaluate the presence of contaminants released from the polymer, as well as its ability to migrate into food simulants. Molar mass (MM) and molar mass distribution are the most important parameters to evaluate the characteristics of the recycled polymer. Therefore, molecular changes were evaluated by rheometry in an oscillatory regimen. The main contribution of the present work was to study the extractability of residual contaminants after going through the recycling process, as well as its impact on the molar mass. In this way, MM distribution in recycled polypropylene for food contact will be further investigated.

\section{2) METHODOLOGY}

The experiment was developed into three main steps as follows: step 1 consists of contaminating the PP with a cocktail of surrogates and recycling it; in step 2, the efficiency of the recycling process was evaluated by an extractability test using different food simulants; in step 3, given the presence of contaminants, the molecular structure was assessed by rheometry after the recycling process.

\section{1) Materials}

Polypropylene in pellets form (Prism 2400) supplied by Braskem S.A, Brazil was used in this project. It presents a melt flow index (MFI) of $20 \mathrm{~g} / 10 \mathrm{~min}$ (ASTM 1238, $230^{\circ} \mathrm{C}, 2.16 \mathrm{~kg}$ ) and density of $0.902 \mathrm{~g} / \mathrm{cm}^{3}$ (ASTM D 792). It is used for food packaging, such as transparent bottles for mineral water, teas and juices. The chemicals used for 
100 the contamination were chloroform (Vetec, 99.8\%, CAS No 67663), toluene (Vetec, 101 99.5\%, CAS No. 108883), benzophenone (Acros Organics, 99\%, CAS No. 119619), 102 tetracosane (Merck, 99\%, CAS No 646311) and heptane (Synth, 99\%, CAS No. 1422825). 103 Ethanol (Merck, 99\%) and acetic acid (Merck, 99\%) were employed to prepare the food 104 simulants, with 10\% (v/v) ethanol and 3\% (w/v) acetic acid respectively in MiliQ water.

\subsubsection{Contamination and recycling (Step 1)}

protocol [20]. The contamination cocktail represents the worst post-consumer condition that the polymer might be exposed to. The contaminants, concentration (volume/volume or mass/volume) and physicochemical properties used were in Table 1.

Table 1: Concentration and physicochemical properties of the surrogates.

\begin{tabular}{|l|c|c|c|}
\hline Contaminants & Concentration & Physicochemical & Vapor pressure (hPa) \\
\hline Benzophenone & $1 \%^{\mathrm{a}}$ & Non-volatile and polar & 1.33 \\
\hline Chloroform & $10 \%^{\mathrm{b}}$ & Volatile and polar & 210 \\
\hline Heptane & $78 \%^{\mathrm{b}}$ & Volatile and non-polar & 111 \\
\hline Toluene & $10 \%^{\mathrm{b}}$ & Volatile and non-polar & 30.88 \\
\hline Tetracosane & $1 \%^{\mathrm{a}}$ & Non-volatile and non-polar & - \\
\hline
\end{tabular}

${ }^{a}$ is $\mathrm{m} / \mathrm{v}$ and $^{b}$ is $\mathrm{v} / \mathrm{v}$. 

three washing stages: (a) 10 minutes with $1 \mathrm{~L}$ of distilled water, (b) $1 \mathrm{~L}$ of $1 \% \mathrm{NaOH}$ solution for 5 minutes and, (c) $1 \mathrm{~L}$ of distilled water finishing with air drying at a temperature of $25^{\circ} \mathrm{C}$. This methodology is well established by Garcia et al [9]. extruder at three extrusion temperatures of 180,190 and $210^{\circ} \mathrm{C}$. The screw speed was maintained at $30 \mathrm{rpm}$ and the pelletizing process was carried out. Table 1 shows the correlation between the samples and their respective nomenclatures after the PP pellets were submitted to the previously mentioned processes.

Table 1: Nomenclature of the PP samples employed in this work.

\begin{tabular}{|l|c|}
\hline Samples & Nomenclature \\
\hline Pristine (unused) PP & PPp \\
\hline Pristine-reprocessed PP & PPpr \\
\hline Contaminated PP & PPc \\
\hline Contaminated-reprocessed PP & PPcr \\
\hline Contaminated-washed PP & PPcw \\
\hline Contaminated-washed-reprocessed PP & PPcwr \\
\hline
\end{tabular}

\subsubsection{Extractability tests (Step 2)}



and ethanol $10 \%$, employed as food simulants. The mixture was hermetically sealed in glass vials and maintained in different conditions: (1) 10 days at $40^{\circ} \mathrm{C},(2) 10$ days at $60^{\circ} \mathrm{C}$ and (3) 2 hours at $70^{\circ} \mathrm{C}$. After the extractability tests, the solutions were filtered, and the surrogates in the simulants were extracted using solid-phase microextraction (SPME) and analyzed by gas chromatography coupled to the mass spectrometer (GC-MS). immersed in the samples for $15 \mathrm{~min}$ at $60^{\circ} \mathrm{C}$ for acetic acid $3 \%$ and $30 \mathrm{~min}$ at $70^{\circ} \mathrm{C}$ for ethanol $10 \%$. Analytes were desorbed in GC $6890 \mathrm{~N}$ gas chromatograph from an Agilent (Palo Alto, CA, USA) injection port for 2 min. A CTC Analytics CombiPal from CTC Analytics AG (Zwingen, Switzerland) was coupled to the gas chromatograph. An HP-5 MS $(30 \mathrm{~cm}$ $\times 0.25 \mathrm{~cm} \times 0.25 \mu \mathrm{m})$ capillary column was used with the following temperature program: start at $40^{\circ} \mathrm{C}$ for $5 \mathrm{~min}$ and increase $10^{\circ} \mathrm{C} \mathrm{min}^{-1}$ up to $300^{\circ} \mathrm{C}$ for $1 \mathrm{~min}$. The injector had a temperature of $250^{\circ} \mathrm{C}$ and helium was the carrier gas used with a flow of $1 \mathrm{~mL} \mathrm{~min}{ }^{-1}$. An Agilent 5975 Mass Spectrometer was used as the detector. The temperatures of the MS source and the quadrupoles were $230^{\circ} \mathrm{C}$ and $250^{\circ} \mathrm{C}$ respectively.

The SCAN mode was applied with a mass range of 50 to $400 \mathrm{~m} / \mathrm{z}$. The compounds were detected from the chromatograms obtained and identified using the mass spectra library (US National Institute of Standards and Technology, NIST) [21]. solutions were prepared in 3\% acetic acid and $10 \%$ ethanol. The following analytical parameters of the applied method were determined: linearity, linear range and limits of detection (LOD) and quantification (LOQ). The signal-to-noise method was applied to 
determine LOD and LIQ. Chromatograms of the analytes at a low concentration were applied to determine the signal-to-noise value.

\subsubsection{Molecular changes (Step 3)}

The complex viscosity $\left(\eta^{*}\right)$, the storage modulus $\left(G^{\prime}\right)$ and the loss modulus ( $\left.G^{\prime \prime}\right)$ as a function of frequency $(\omega)$ were determined by a parallel plate rheometer (Anton Paar MCR 305). The tests were performed at $190^{\circ} \mathrm{C}$ in oscillatory mode. The parameters used were: $25 \mathrm{~mm}$ diameter plates, $1 \mathrm{~mm}$ gap, and the range of frequency used was 0.1 to $500 \mathrm{rad} / \mathrm{s}$ at $1 \%$ strain, which proved to be in the linear viscoelastic range according to a prior amplitude sweep test.

\section{3) RESULTS AND DISCUSSION}

\section{1) Migration of contaminants}

The analytical parameters are shown in supplementary material. Linear ranges from $0.0002 \mathrm{mg} \mathrm{kg}^{-1}$ to $4735 \mathrm{mg} \mathrm{kg}^{-1}$ with regression coefficients $(r)$ in the range of 0.9903 to 0.9997 were observed. The lowest values of LOD, indicating sensitivity of the method, were obtained for toluene in both solvents $3 \%$ acetic acid (LOD $=0.00006 \mathrm{mg}$ $\left.\mathrm{kg}^{-1}\right)$ and $10 \%$ ethanol $\left(\mathrm{LOD}=0.00005 \mathrm{mg} \mathrm{kg}^{-1}\right)$. In contrast, the highest LOD was obtained for tetracosane in $3 \%$ acetic acid $\left(\mathrm{LOD}=0.0088 \mathrm{mg} \mathrm{kg}^{-1}\right)$ and heptane in $10 \%$ ethanol $\left(\mathrm{LOD}=0.0031 \mathrm{mg} \mathrm{kg}^{-1}\right)$. The same tendency was noticed for LOQ values 

analyzed by extractability tests since pellets instead of bottles or containers were

177 available for the study. Therefore, it was possible to identify the influence of recycling on the decontamination process. Figure $1(a, b, c, d$ and e) presents the results of the 179 extractability for toluene, tetracosane, chloroform and benzophenone in acetic acid 3\% and ethanol $10 \%$ as food simulants.

a)

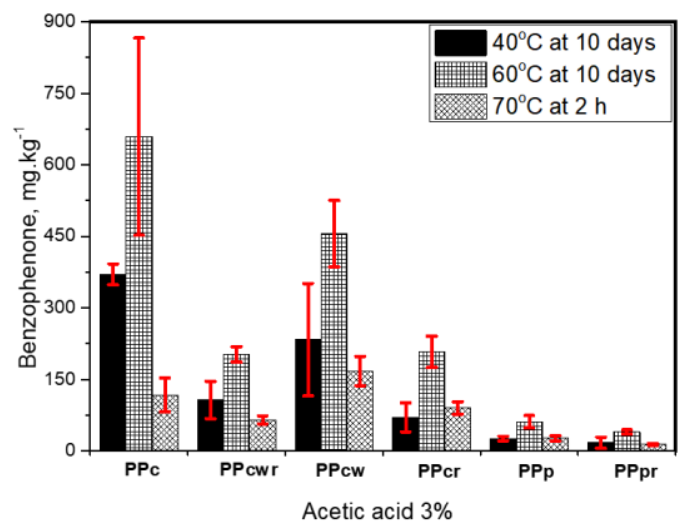

181

b)

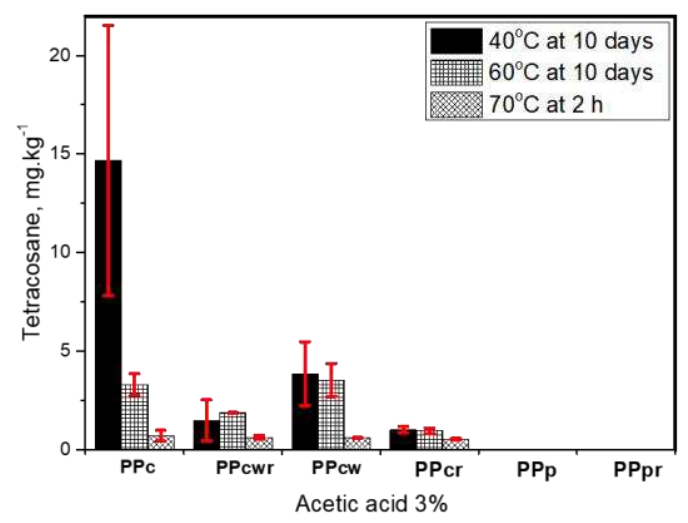

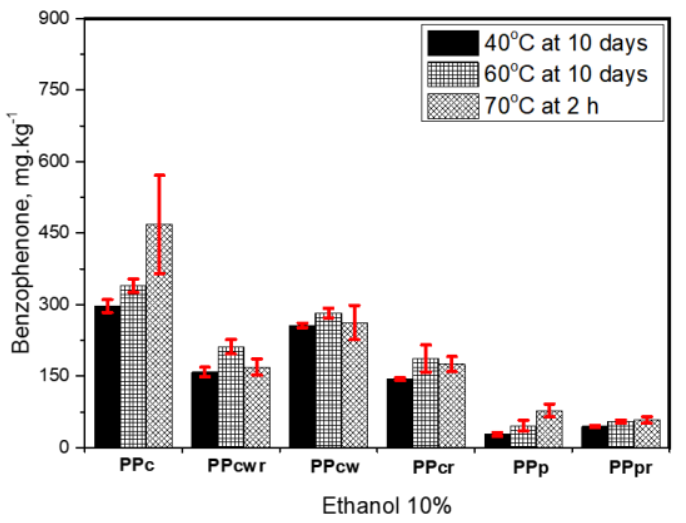

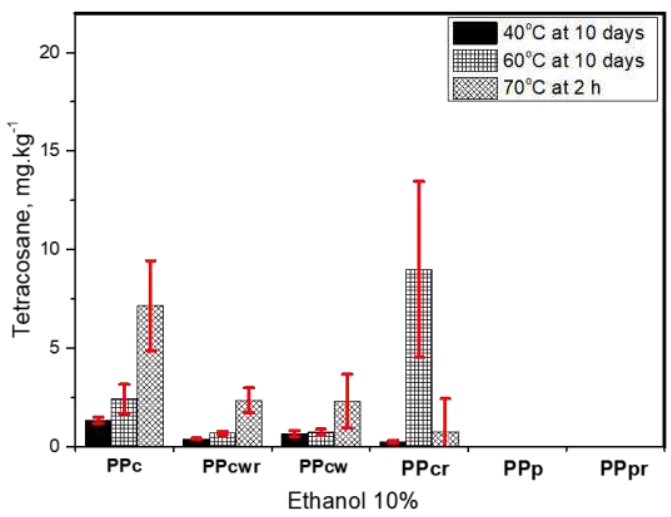


c)

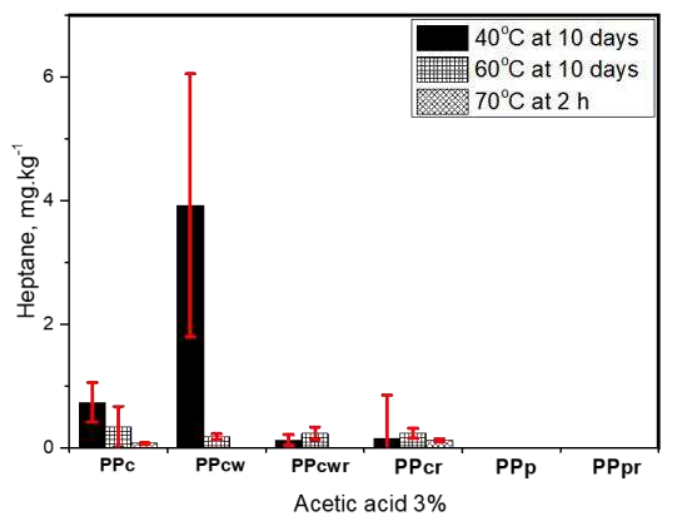

183

d)

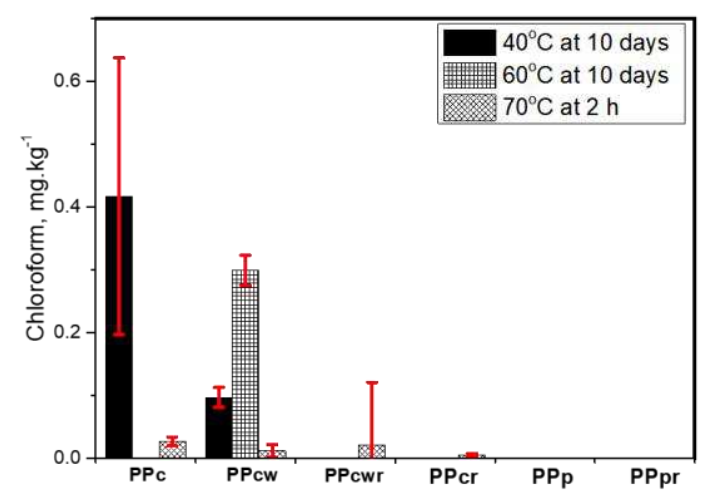

(a) Acetic acid $3 \%$
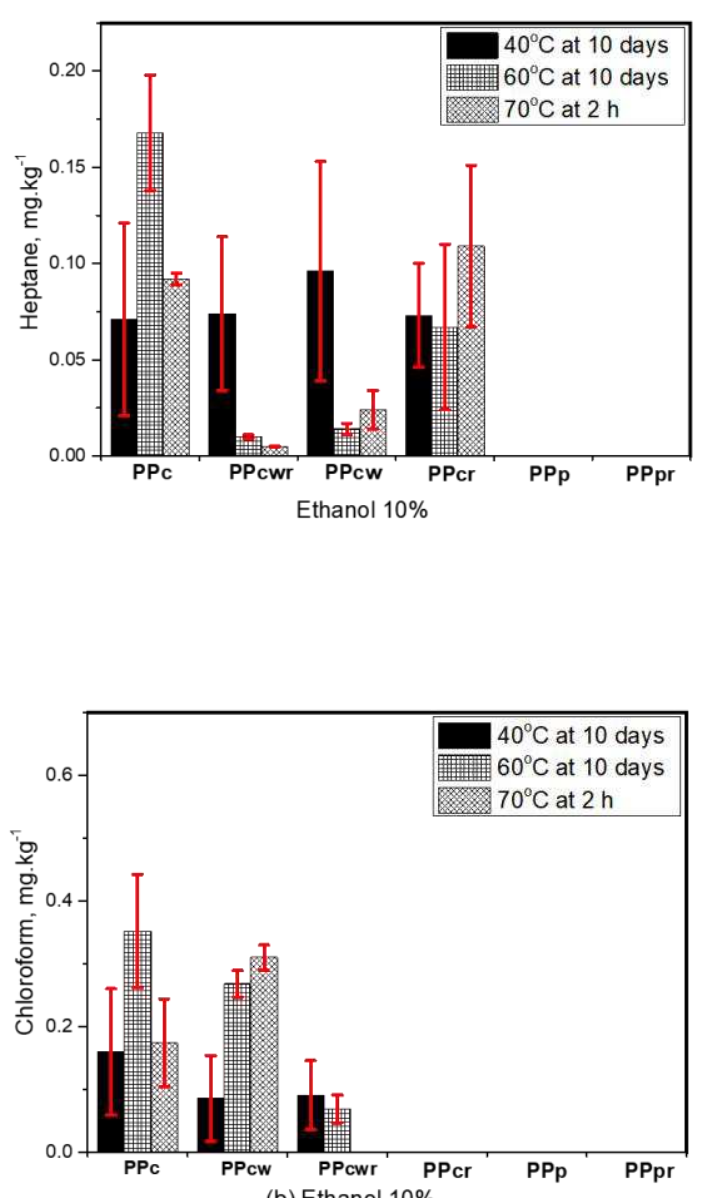

(b) Ethanol $10 \%$

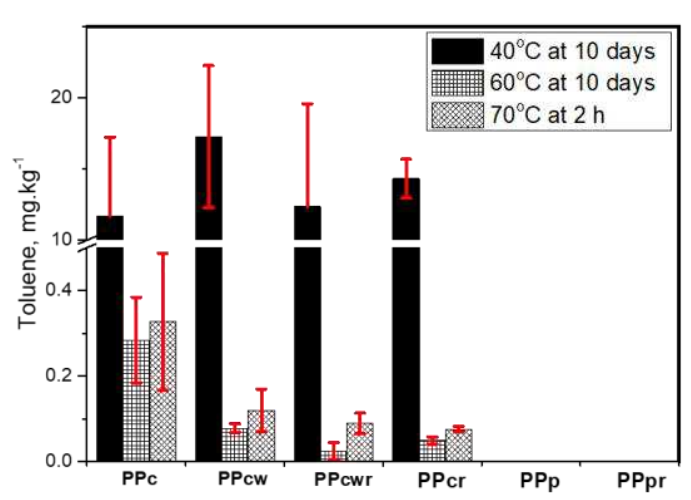

(b) Ethanol $10 \%$

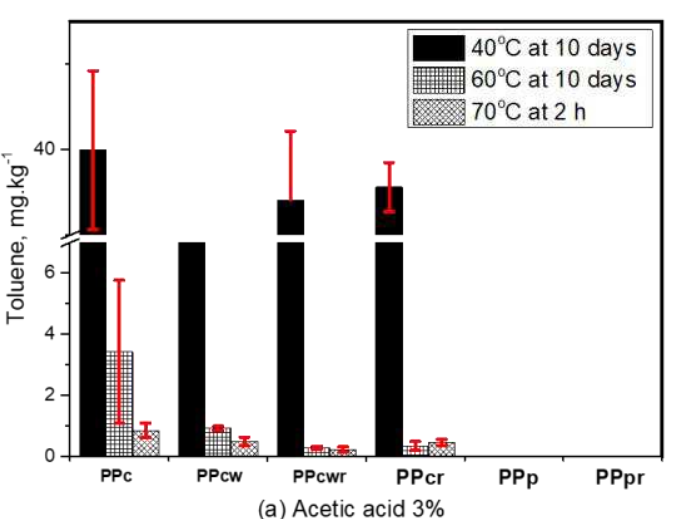

186 Figure 1: Results of the extractability test on the PP samples for (a) benzophenone, (b) days and $70^{\circ} \mathrm{C}$ at $2 \mathrm{~h}$. 

processes and all of them may occur in the step of contamination as well as during the extractability test. Additionally, the time, temperature, physicochemical properties of the surrogate and molecular characteristics of the polymer impact these processes. Consequently, this is a complex system and its workability depends on intrinsic and extrinsic factors and its relationship with the characteristics of each polymer. are not present in the PPp and PPpr, with exception of benzophenone. This contaminant is considered a non-intentionally added substance (NIAS), once it is widely used as a photoinitiator in the polymerization process. A recent study regarding the identification of different volatile compounds and odoriferous compounds present in recycled polypropylene carried out by Paiva et al.[23], showed that benzophenone is present in all studied samples, including pristine polypropylene. and temperature, the results present in Figure 1 show that these parameters are not the most important for this system. For example, the molar mass of the surrogates increases in the following order: toluene $\left(92.14 \mathrm{~g} / \mathrm{cm}^{3}\right)>$ chloroform $\left(119.38 \mathrm{~g} / \mathrm{cm}^{3}\right)>$ benzophenone $\left(182.2 \mathrm{~g} / \mathrm{cm}^{3}\right)>$ tetracosane $\left(338.65 \mathrm{~g} / \mathrm{cm}^{3}\right)$. Nevertheless, despite this observation, there was no correlation between the molar mass of the surrogates and the extractability (Figure 1). Therefore, the molar mass does not seem to be an important parameter in this case. 
understanding the diffusion process. It would be expected that the rate of diffusion

213 increases with the temperature, as described by Voultzatis et al. [24]. Based on this

214 consideration, the diffusion of surrogates was expected to be faster at $70^{\circ} \mathrm{C}$ and slower at $60^{\circ} \mathrm{C}$, considering the same time (10 days). The glass transition temperature was significantly lower for the PP $[25]\left(\mathrm{Tg} \cong-20^{\circ} \mathrm{C}\right)$, which means that at the experimental temperature, the PP is in a rubbery state $(\mathrm{T}>\mathrm{Tg})$. It consists of a liquid-like structure with high segmental motions resulting in an increase of free volume as a function of temperature. This behavior explains the greater diffusivity in polyolefins, such as PP and PE compared to PET ( $\left.\mathrm{Tg} \cong 69^{\circ} \mathrm{C}\right)$, as described by Palkopoulou et al. [26]. However, the influence of temperature is not linear for all contaminants since it was observed, for example, that tetracosane and chloroform migrated more at $40^{\circ} \mathrm{C}$ in $3 \%$ acetic acid than in $10 \%$ ethanol. In most cases, time is a crucial parameter for migration, since the diffusion process depends on it as previously mentioned. The highest migration was observed for benzophenone. Benzophenone is a polar, non-volatile compound with a chemical group with free electrons in the oxygen that can strongly interact with the oxygen in $3 \%$ acetic acid and $10 \%$ ethanol [27].

Contrarily, chloroform (a polar and volatile compound) presents low concentration, as expected. Consequently, it is impossible to detect it in the PPcr sample, according to LOD and LOQ present in Table 2. The chloroform presents a high vapor pressure, which is a parameter that controls the volatility of a chemical, and the temperature of measurements $\left(40,60\right.$ and $\left.70^{\circ} \mathrm{C}\right)$ may contribute to low values of concentration of these surrogates. 
toluene is lower since the chemical affinity with polymer (non-polar) is high. Considering

236 that the system consists of polymer, food simulant and surrogate, chemical similarity

237 plays an important role in this process. Both simulants present similar polarity [28] but

238 toluene presents a lower molar mass $(92.10 \mathrm{~g} / \mathrm{mol})$ when compared to tetracosane

239 (338.65 g/mol). As described previously, the molar mass affects the diffusion process,

240 i.e., the higher the molar mass the lower the diffusion of contaminants. The chemical similarity between polymer and surrogates explains the low concentration in food simulants for non-polar surrogates. The opposite results were obtained by Oliveira et al.[27] using PET as the matrix and toluene as the contaminant. PET is a polar polymer and, according to the authors, the high concentration of these contaminants was due to the low affinity with this polymer.

\section{2) Influence of recycling on the migration}

The effects of decontamination were verified and the obtained results were analyzed considering each step (washing and reprocessing). There are several different types of washing processes for post-consumer polymers. They are very dependent on the available technology, as well as, the final destination of the product [29]. The cleaning process for the food market is done in critical and extreme conditions to guarantee high levels of decontamination [16]. In this work, we decided to use a common process to clean recycled resin. In all cases, the cleaning process helps to decontaminate the samples. This behavior is clear when the PPc is compared with the PPcw. 

submitted to an extrusion process present a lower level of contaminants. First of all, during the extrusion process, the polymer is melting and in the melting state, the diffusion process increases significantly [16] and enhances the decontamination, especially that of volatile compounds because of the high temperature. Additionally, during the reprocessing, the polymer is continuously submitted to a high shear rate and temperature. In both cases, the mobility of polymer chains and that of the molecules of contaminants is favorable. Although technologies of PET decontamination are widely investigated $[2,30,31]$, literature is scarce when it comes to PP. One exception is the work developed by Garcia, Cruz and Nerin [15] that analyzed different extrusion processes during the decontamination of the PP samples. The obtained results showed that the most efficient process was the co-rotating twin-screw extruder with forced degassing, which was attributed to the higher shear rates and the degassing system.

\section{3) Influence of residual contaminants into the molecular structure}

The quality, aggregate value and employability of the recycled polymers are associated with the degradation degree that polymers may suffer after the recycling process. Two aspects must be considered when a polymer is submitted to high shear rates, temperature and presence of contaminants: (i) the chain scission and thus, its molar mass, which is reflected in mechanical and thermal properties, and (ii) the flow characteristics, which affects the processability. Although this is an important aspect, 
Table 3: Crossover point values, $G^{\prime}(\omega)=G^{\prime \prime}(\omega)$, for the samples.

\begin{tabular}{|l|c|c|}
\cline { 2 - 3 } \multicolumn{1}{c|}{} & $\mathrm{G}^{\prime}(\omega)=\mathrm{G}^{\prime \prime}(\omega)$ & $\omega(\mathrm{rad} / \mathrm{s})$ \\
\hline PPp & 29072 & 84 \\
\hline PPpr & 29409 & 92 \\
\hline PPc & 30189 & 95 \\
\hline PPcr & 36940 & 302 \\
\hline PPcw & 31728 & 121 \\
\hline PPcwr & 33892 & 164 \\
\hline
\end{tabular}
molar mass. This effect was clearly observed when the samples without contaminants (PPp, PPpr) were compared with their contaminated counterparts (PPc, PPcr). The samples submitted to reprocessing in the presence of contaminants (PPcr and PPcwr) showed higher levels of degradation, and, consequently, lower values of molar mass. 
296 The presence of contaminants together with high shear rates and temperature, 297 characteristics of the extrusion process, lead to a scission of the PP chain. The 298 degradation of PP occurs by a radical scission chain, especially in the tertiary carbon [34]. 299 The formation of free macroradicals occurs in the initiation phase, after which these 300 radicals react with oxygen forming peroxy radicals. In the propagation phase of the 301 reactions, the peroxy radicals abstract the hydrogens from the tertiary polypropylene carbons to form the hydroperoxides and more free radicals. In the termination phase, free radicals bind and there is also the formation of carbonyl groups, a typical characteristic of the $\beta$ scission of the polypropylene chain [34]. Another important parameter, that was analyzed, is the molar mass distribution (MMD). The PP degradation leads to narrowing the MMD, which means that the lower values of molar mass presented a significant narrowing in the MMD [35-39]. Incarnato et al. conducted a study about the effect of reprocessing steps on the physical and molecular properties of recycled polypropylene. The results of the intrinsic viscosity analysis showed that there was a decrease in molar mass and a narrowing distribution of molar mass compared to pristine polypropylene [37]. Figure 3. In the samples that present a high level of degradation (PPcr), a higher Newtonian plateau can be observed. On the other hand, in the samples with higher molar mass and widened MMD the plateau is significantly lower due to the inferior chain length that can act as a lubricant, increasing the pseudoplastic behavior of the sample. 


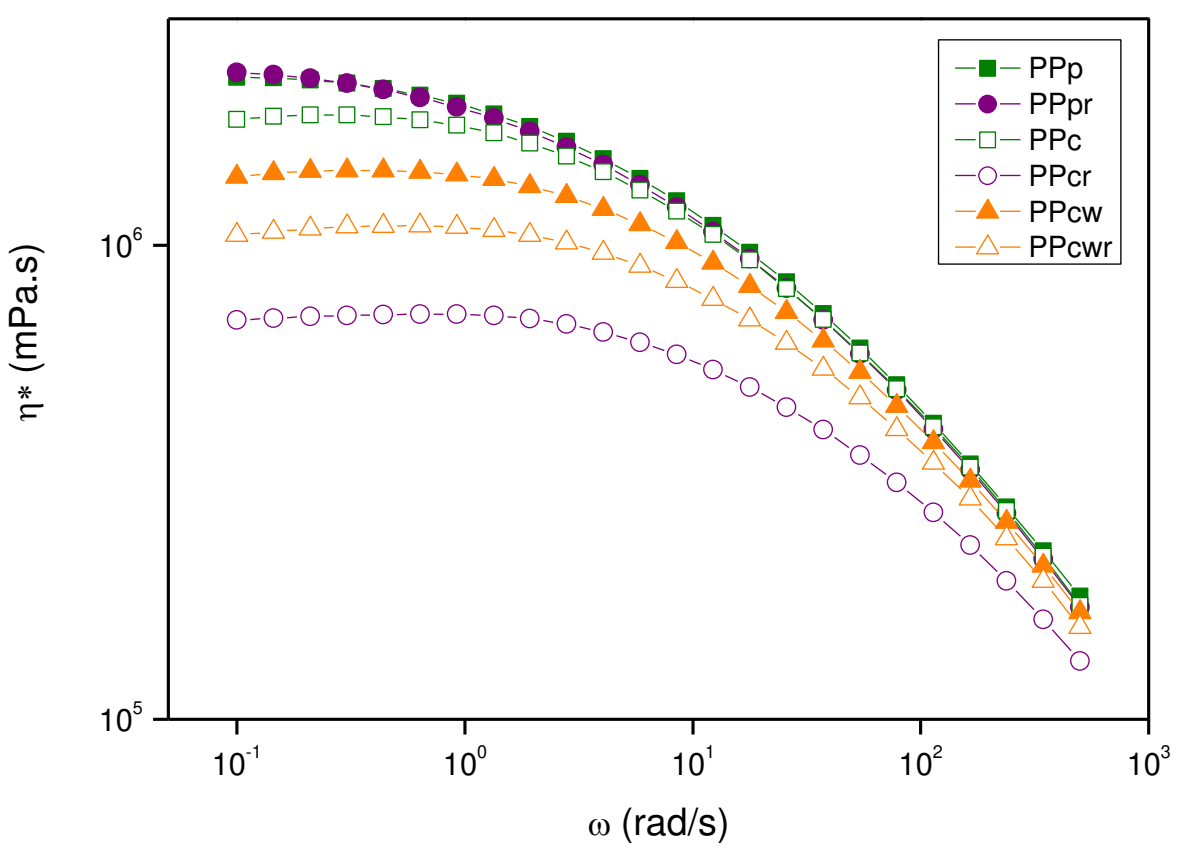

317

318 Figure 3: Complex frequency as a function of frequency for all samples.

Figure 4 depicts the results of the storage modulus $\left(G^{\prime}\right)$ and the loss modulus

$321\left(G^{\prime \prime}\right)$ as a function of the frequency. These parameters are dependent on the length and 322 the level of the entanglements of the chains. The samples with a higher level of 323 degradation show lower values of $G^{\prime}$ and $G^{\prime \prime}$. These results confirm those previously obtained, indicating that the contaminants together with reprocessing significantly decrease the molar mass and the level of entanglements. 

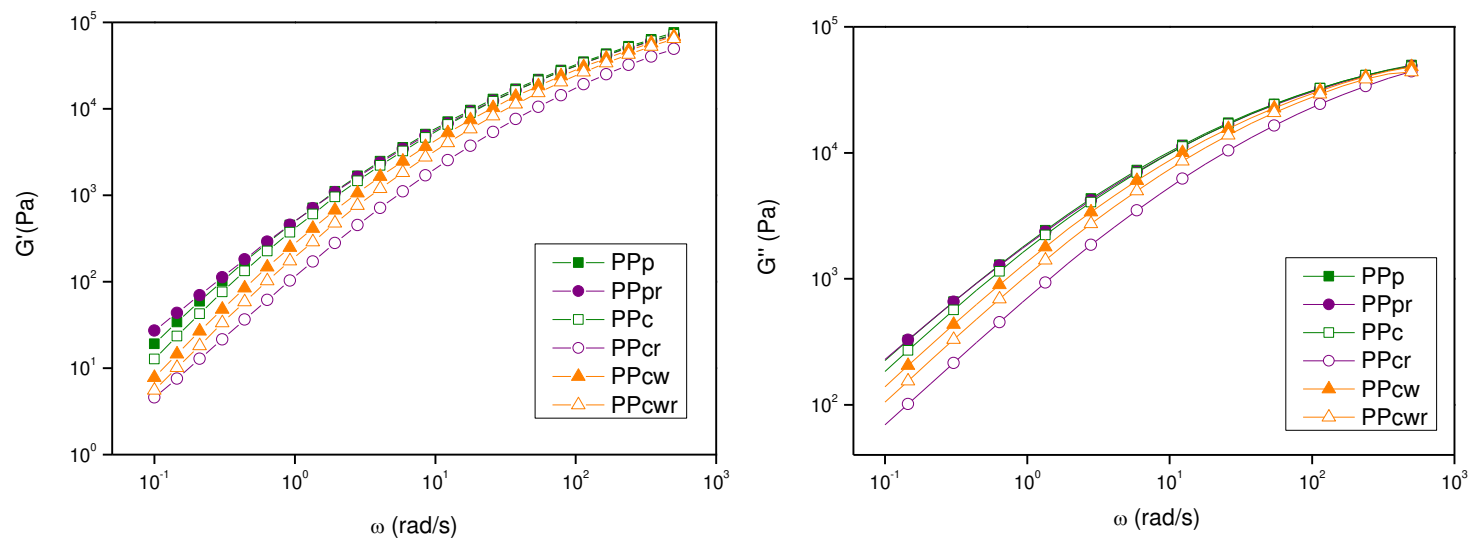

(a)

327

328

329

330

331

332

333

334

335

Figure 4: Storage modulus $\left(G^{\prime}\right)$ as a function of the frequency for all samples (a) and loss modulus $\left(G^{\prime \prime}\right)$ as a function of the frequency for all samples (b).

Additionally, an interesting behavior has been observed when the extractability measurements were compared to rheometry. First, the samples with a lower level of contaminants presented the higher molar mass (PPp and PPpr). Nevertheless, the contaminants alone have not been able to decrease the molar mass significantly (PPc), even when surrogates were present at high levels (Figure 2). The samples extruded with a high content of contaminants (PPcr) have lower molar mass. These results prove that the higher temperatures and shear rates, characteristics of the extrusion process, together with contaminants are the key points to understanding the molecular structure changes of the recycled polymer. Additionally, the reprocessing and cleaning process decreased the level of contaminants. Therefore, it is an important issue to make feasible the use of recycled material for direct contact with food. 
This work addressed the extractability of residual contaminants after going through the recycling process, as well as its impact on the polypropylene molecular structure. This was associated with the packaging misuse that leads to the contamination of the polymer with chemical products, which will eventually migrate into food or decrease the molar mass making its applicability unfeasible.

The extractability results showed that there was no correlation between the molar mass of the surrogates and employed temperature. On the other hand, the chemical affinity between the surrogate and food plays an important role in this complex process. Almost all contaminants were not present in the PPp and PPpr, with exception of benzophenone. It should be highlighted that this contaminant is considered as an intentionally added substance, once it is widely used as a photoinitiator in the polymerization process. Chloroform presented high vapor pressure and it was determined to have a low concentration. Regarding the non-polar surrogates, the concentration of tetracosane and toluene was low, since its chemical affinity with the polymer (non-polar) was high.

decontamination process (comparing PPcw to PPcwr and PPc to PPcr) due to the high temperatures and shear rates. For all surrogates, their quantified concentration was significantly lower after the washing and extrusion processes. that they may suffer after the recycling process. It may affect its molar mass, which not only impacts its properties but also its flow characteristics, which affects the 
processability. Rheometry results showed that the presence of contaminants affected the PP molar mass. This effect was more significant when the samples were submitted to reprocessing in the presence of surrogates. Also, the samples with higher molar mass and widening MMD presented a significant lower Newtonian plateau, due to the inferior chain length that can act as a lubricant increasing the pseudoplastic behavior of the sample. These results prove that the high temperatures and shear rates, characteristics of the extrusion process, together with contaminants are the keys to understanding the molecular structure changes of the recycled polymer. Notwithstanding, in some cases, residual levels of some surrogates above those permitted by the legislation were still detected in the resin even after the recycling process.

\section{Acknowledgments}

The authors are thankful for the financial and the São Paulo Research Foundation (FAPESP) for grants 2017/23659-9 and 2016/25703-2.

\section{Conflict of Interest}

The authors confirm that they have no conflicts of interest with respect to the work described in this manuscript.

\section{Availability of data and material}


[1] J.N. Hahladakis, C.A. Velis, R. Weber, E. lacovidou, P. Purnell, An overview of chemical additives present in plastics: Migration, release, fate and environmental impact during their use, disposal and recycling, J. Hazard. Mater. 344 (2018) 179199. https://doi.org/10.1016/j.jhazmat.2017.10.014.

[2] C. Dutra, M.T.D.A. Freire, C. Nerín, K. Bentayeb, A. Rodriguez-Lafuente, M. Aznar, F.G.R. Reyes, Migration of residual nonvolatile and inorganic compounds from recycled post-consumer PET and HDPE, J. Braz. Chem. Soc. 25 (2014) 686-696. https://doi.org/10.5935/0103-5053.20140016.

[3] US Food and Drug Administration (FDA), Guidance for industry: Use of recycled plastics in food packaging, Chem. Considerations. HFS-275. Washington, DC US FDA, Cent. Food Saf. Appl. Nutr. 4. (n.d.).

[4] COMMISSION REGULATION (EU), COMMISSION REGULATION (EU) No 10/2011 of 14 January 2011 on plastic materials and articles intended to come into contact with food., 2011.

[5] ANVISA, Resolução-DOU $n^{\circ}$ - 228, de 30 de novembro de 2010. Regulamento técnico MERCOSUL sobre migração em materiais, embalagens e equipamentos plásticos destinados a entrar em contato com alimentos., Diário Of. Da União, Brasília, DF. 1 (2010) 105. https://doi.org/10.1017/CBO9781107415324.004.

[6] C. Direto, Legislação de embalagem para contato com alimentos: MERCOSUL e outros países Latinoamericados, Polímeros. 14 (2004) E8-E13. https://doi.org/10.1590/s0104-14282004000100004.

[7] C. Munoz, A. Eicher, M. Biedermann, K. Grob, Recycled paperboard with a barrier layer for food contact: set-off during stacking or reeling. Analytical method and preliminary results, Food Addit. Contam. - Part A Chem. Anal. Control. Expo. Risk Assess. 35 (2018) 577-582. https://doi.org/10.1080/19440049.2017.1411618.

[8] C. Nerín, R. Batlle, Assessing the suitability of recycled plastics used as agricultural soil covers: Migration study and experimental harvest, J. Agric. Food Chem. 47 (1999) 285-293. https://doi.org/10.1021/jf9804950. 
[9] P.S. Garcia, S.A. Cruz, C. Nerín, Comparison of different extrusion processes for cleaning the recycled polypropylene removing volatile and non-volatile contaminants, Prog. Rubber, Plast. Recycl. Technol. 30 (2014) 37-54. https://doi.org/10.1177/147776061403000103.

[10] Z.F. Chen, Q.B. Lin, X.C. Song, S. Chen, H.N. Zhong, C. Nerin, Discrimination of Virgin and Recycled Polyethylene Based on Volatile Organic Compounds Using a Headspace GC-MS Coupled with Chemometrics Approach, Food Packag. Shelf Life. 26 (2020) 100553. https://doi.org/10.1016/j.fpsl.2020.100553.

[11] Q.-Z. Su, P. Vera, C. Nerín, Direct Immersion-Solid-Phase Microextraction Coupled to Gas Chromatography-Mass Spectrometry and Response Surface Methodology for Nontarget Screening of (Semi-) Volatile Migrants from Food Contact Materials, Anal. Chem. 92 (2020) 5577-5584. https://doi.org/10.1021/acs.analchem.0c00532.

[12] P. Dole, A.E. Feigenbaum, C. De La Cruz, S. Pastorelli, P. Paseiro, T. Hankemeier, Y. Voulzatis, S. Aucejo, P. Saillard, C. Papaspyrides, Typical diffusion behaviour in packaging polymers - Application to functional barriers, Food Addit. Contam. 23 (2006) 202-211. https://doi.org/10.1080/02652030500373661.

[13] PlasticsEurope, Risk assessment of non-listed substances (NLS) and nonintetionally added substances (NIAS) under article 19, 32 (2011).

[14] B. Geueke, K. Groh, J. Muncke, Food packaging in the circular economy: Overview of chemical safety aspects for commonly used materials, J. Clean. Prod. 193 (2018) 491-505. https://doi.org/10.1016/j.jclepro.2018.05.005.

[15] P.S. Garcia, C.H. Scuracchio, S.A. Cruz, Effect of residual contaminants and of different types of extrusion processes on the rheological properties of the postconsumer polypropylene, Polym. Test. 32 (2013) 1237-1243. https://doi.org/10.1016/j.polymertesting.2013.08.002.

[16] S. Palkopoulou, C. Joly, A. Feigenbaum, C.D. Papaspyrides, P. Dole, Critical review on challenge tests to demonstrate decontamination of polyolefins intended for food contact applications, Trends Food Sci. Technol. 49 (2016) 110-120. https://doi.org/10.1016/j.tifs.2015.12.003. 
[17] X.C. Song, M. Wrona, C. Nerin, Q.B. Lin, H.N. Zhong, Volatile non-intentionally added substances (NIAS) identified in recycled expanded polystyrene containers and their migration into food simulants, Food Packag. Shelf Life. 20 (2019) 100318. https://doi.org/10.1016/j.fpsl.2019.100318.

[18] L. Coulier, H.G.M. Orbons, R. Rijk, Analytical protocol to study the food safety of (multiple-)recycled high-density polyethylene (HDPE) and polypropylene (PP) crates: Influence of recycling on the migration and formation of degradation products, Polym. Degrad. Stab. $92 \quad$ (2007) 2016-2025. https://doi.org/10.1016/j.polymdegradstab.2007.07.022.

[19] S.A. Cruz, M. Zanin, P.A.P. Nascente, M.A. Bica de Moraes, Superficial modification in recycled PET by plasma etching for food packaging, J. Appl. Polym. Sci. 115 (2010) 2728-2733. https://doi.org/10.1002/app.29958.

[20] US Food and Drug Administration (FDA), Food and Drugs Administration (FDA)., Guid. Ind. Food Contact Subst. Use Recycl. Plast. Food Packag. Chem. Considerations. (2006). https://www.fda.gov/regulatory-information/searchfda-guidance-documents/guidance-industry-use-recycled-plastics-foodpackaging-chemistry-considerations acesso em mar. 2020.

[21] NIST (National Institue of Standards and Technology), Libro del Web de Química del NIST, SRD 69, Retrieved March 26, 2020, from Https//Webbook.Nist.Gov/. (n.d.).

[22] P. Vera, E. Canellas, C. Nerín, Compounds responsible for off-odors in several samples composed by polypropylene, polyethylene, paper and cardboard used as food packaging materials, Food Chem. $309 \quad$ (2020) 125792. https://doi.org/10.1016/j.foodchem.2019.125792.

[23] R. Paiva, M. Wrona, C. Nerín, I. Bertochi, L. Gavril, S. Andrea, Importance of profile of volatile and off-odors compounds from different recycled polypropylene used for food applications, Food Chem. $350 \quad$ (2021) 129250. https://doi.org/10.1016/j.foodchem.2021.129250.

[24] I.S. Voultzatis, C.D. Papaspyrides, C.J. Tsenoglou, C. Roussis, Diffusion of model contaminants in high-density polyethylene, Macromol. Mater. Eng. 292 (2007) 
[25] N. Ramesh, J.L. Duda, Diffusion in Polymers below the Glass Transition Temperature: Comparison of Two Approaches Based on Free Volume Concepts, Korean J. Chem. Eng. 17 (2000) 310-317. https://doi.org/10.1007/BF02699046.

[26] C. Kuswanti, G. Xu, J. Qiao, J.A. Stuart, K. Koelling, B. Lilly, An Engineering Approach to Rheological Characterization, Ind. Eng. 6 (2003).

[27] É.C. Oliveira, S.A. Cruz, P.H.L. Aguiar, Effect of PECVD deposition parameters on

[29] R. Demets, M. Roosen, L. Vandermeersch, K. Ragaert, C. Walgraeve, S. De Meester, Development and application of an analytical method to quantify odour removal in plastic waste recycling processes, Resour. Conserv. Recycl. 161 (2020) 104907. https://doi.org/10.1016/j.resconrec.2020.104907.

[30] S.A. Cruz, C.H. Scuracchio, L.B. Fitaroni, C. Oliveira, The use of melt rheology and solution viscometry for degradation study of post-consumer poly(ethylene terephthalate): The effects of the contaminants, reprocessing and solid state polymerization, Polym. Test. $60 \quad$ (2017) 236-241. https://doi.org/10.1016/j.polymertesting.2017.03.026.

[31] W. Romão, M.A.S. Spinacé, M.A. De Paoli, Poly(ethylene terephthalate), PET: A review on the synthesis processes, degradation mechanisms and its recycling, Polimeros. 19 (2009) 121-132. https://doi.org/10.1590/s010414282009000200009 .

[32] S.A. Cruz, M. Zanin, Evaluation and identification of degradative processes in postconsumer recycled high-density polyethylene, Polym. Degrad. Stab. 80 (2003) 3137. https://doi.org/10.1016/S0141-3910(02)00379-8.

[33] F.L.S. Freitas, A.C. Chinellato, S.A. Cruz, Molar Mass Alteration During Post- 
Consumer PET Recycling Using Polycarbodiimide-Based Additive, J. Polym. Environ. 29 (2021) 734-744. https://doi.org/10.1007/s10924-020-01896-4.

[34] J. a. M. Agnelli, M. a. Chinelatto, Degradação de Polipropileno : Aspectos Teóricos e Recentes Avanços Em Sua Estabilização, Polímeros Ciência e Tecnol. 2 (1992) 27-31.

[35] L.B. Fitaroni, J.A. De Lima, S.A. Cruz, W.R. Waldman, Thermal stability of polypropylene-montmorillonite clay nanocomposites: Limitation of the thermogravimetric analysis, Polym. Degrad. Stab. 111 (2015) 102-108. https://doi.org/10.1016/j.polymdegradstab.2014.10.016.

[36] L. Incarnato, P. Scarfato, D. Acierno, M.R. Milana, R. Feliciani, Influence of recycling and contamination on structure and transport properties of polypropylene, J. Appl. Polym. Sci. 89 (2003) 1768-1778. https://doi.org/10.1002/app.12168.

[37] L. Incarnato, P. Scarfato, D. Acierno, Rheological and mechanical properties of recycled polypropylene, Polym. Eng. Sci. 39 (1999) 749-755. https://doi.org/10.1002/pen.11463.

[38] L. Incarnato, L. Di Maio, D. Aciernot, M. Denaro, L. Arrivabene, Relationships between processing-structure-migration properties for recycled polypropylene in food packaging, Food Addit. Contam. 15 (1998) 195-202. https://doi.org/10.1080/02652039809374630.

[39] R.V. de Camargo, C. Saron, Mechanical-Chemical Recycling of Low-Density Polyethylene Waste with Polypropylene, J. Polym. Environ. 28 (2020) 794-802. https://doi.org/10.1007/s10924-019-01642-5. 
Figures
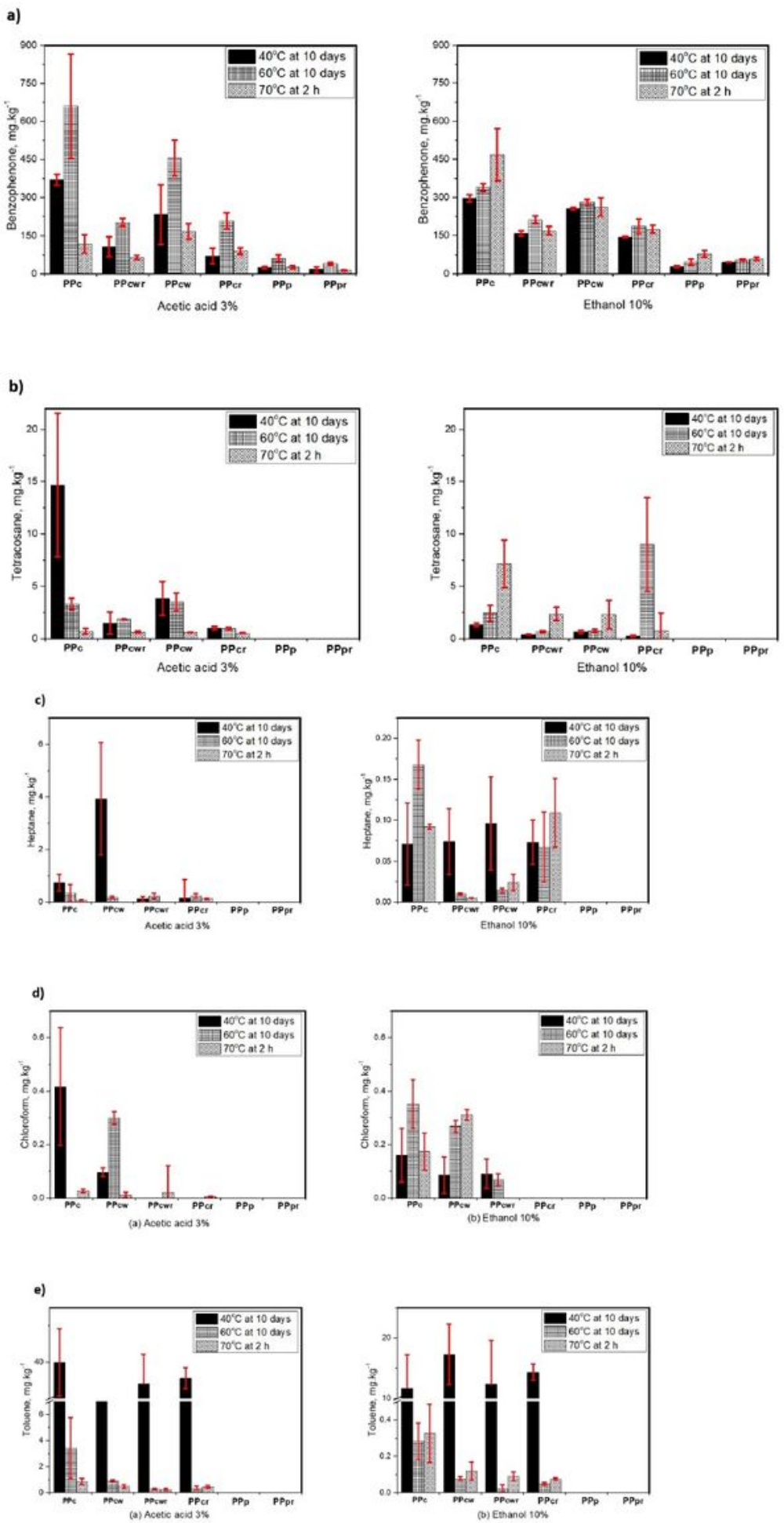

\section{Figure 1}

Results of the extractability test on the PP samples for (a) benzophenone, (b) tetracosane, (c) heptane, (d) chloroform and (e) toluene for $40^{\circ} \mathrm{C}$ at 10 days, $60^{\circ} \mathrm{C}$ at 10 days and $70^{\circ} \mathrm{C}$ at $2 \mathrm{~h}$. 


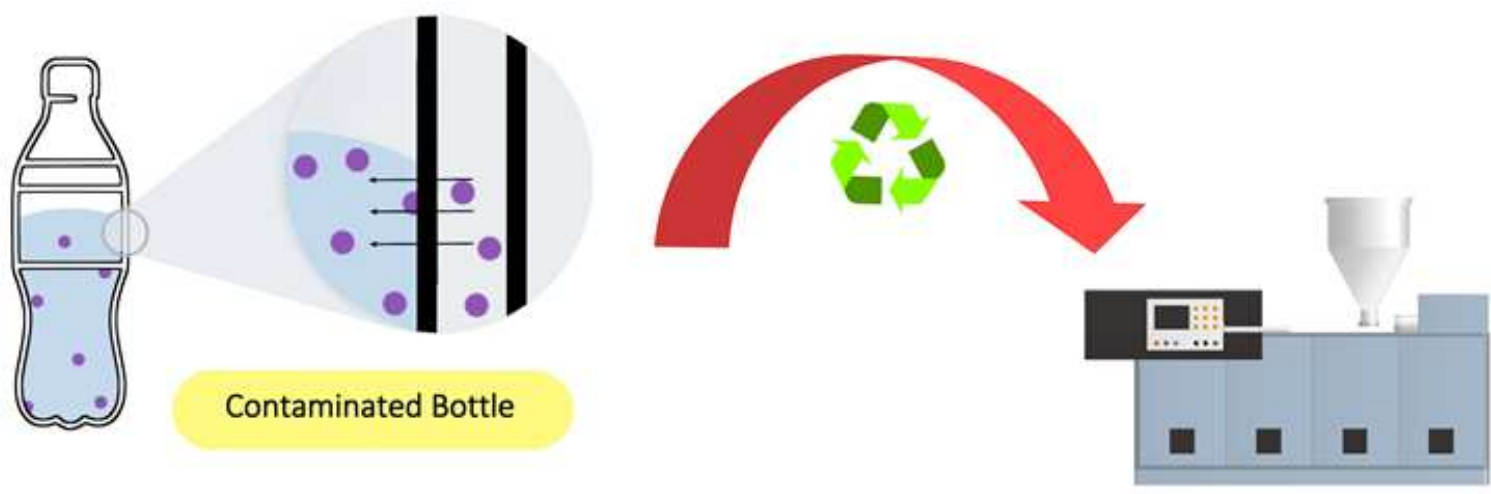

DECONTAMINATION

PARTIAL

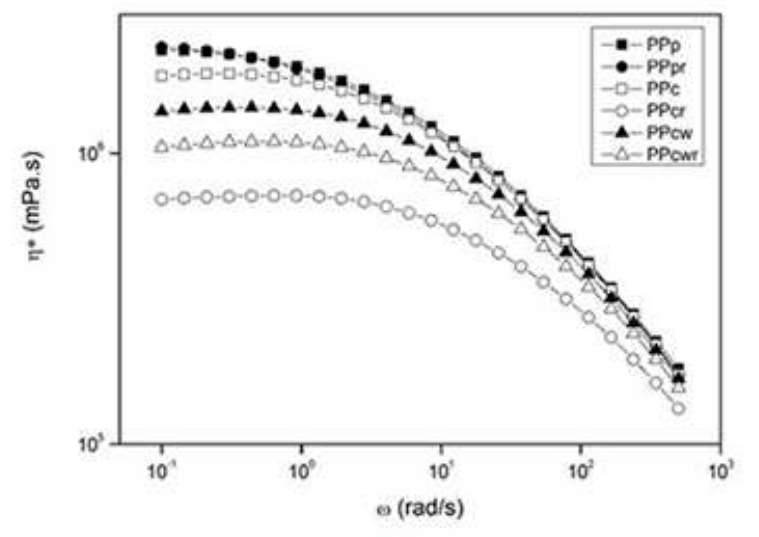

PARTIAL SCISSION OF CHAIN

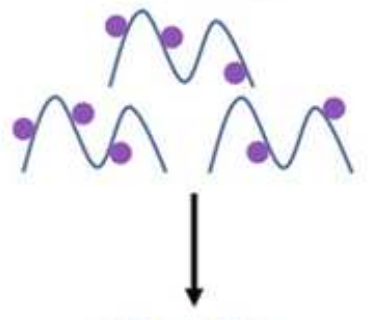

$92 \% p_{m}$

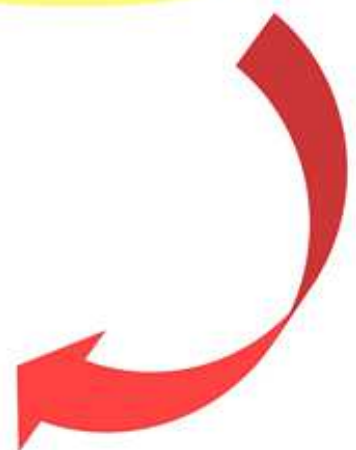

Figure 2

Nevertheless, the contaminants alone have not been able to decrease the molar mass significantly (PPc), even when surrogates were present at high levels (Figure 2). 


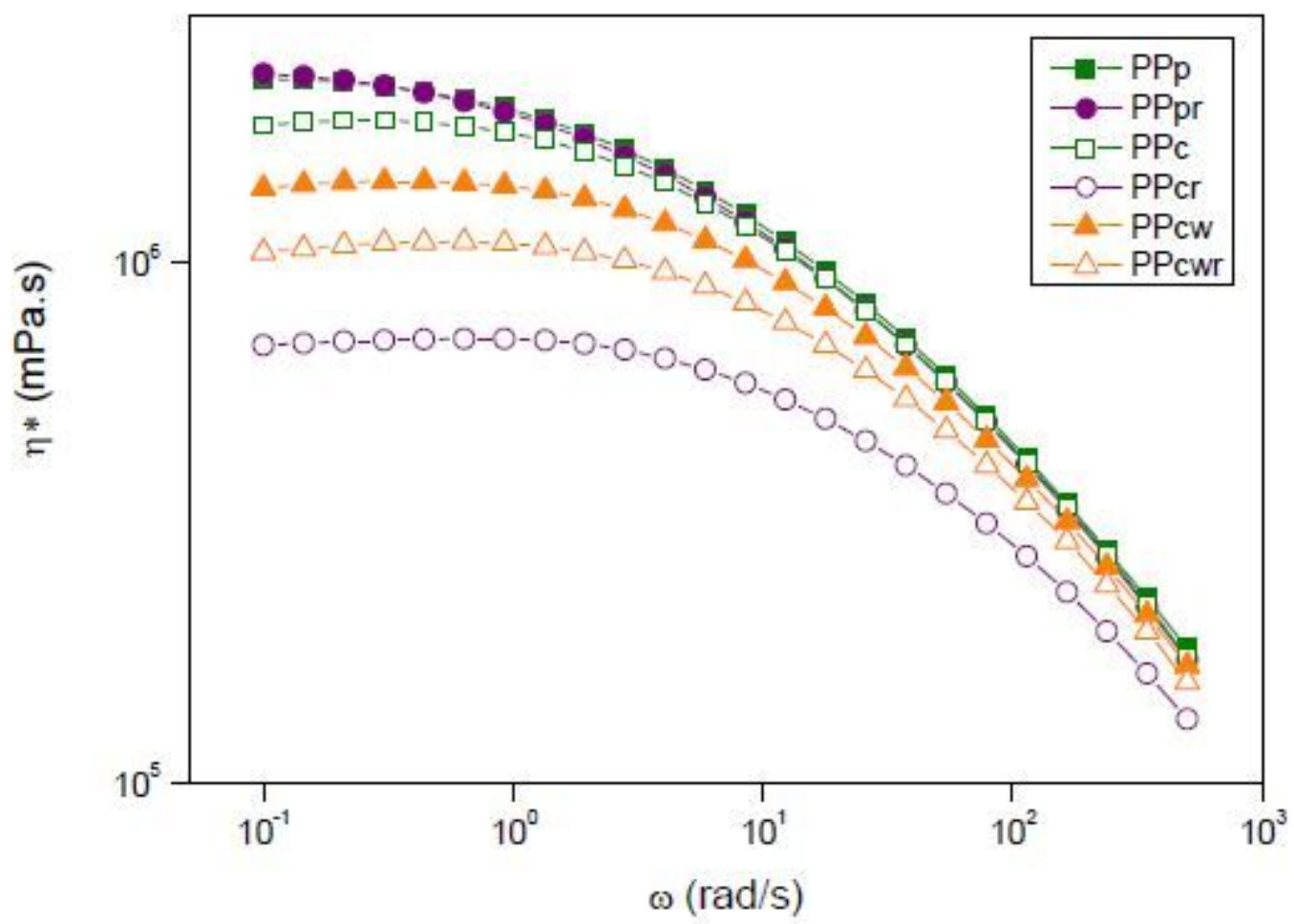

Figure 3

Complex frequency as a function of frequency for all samples.
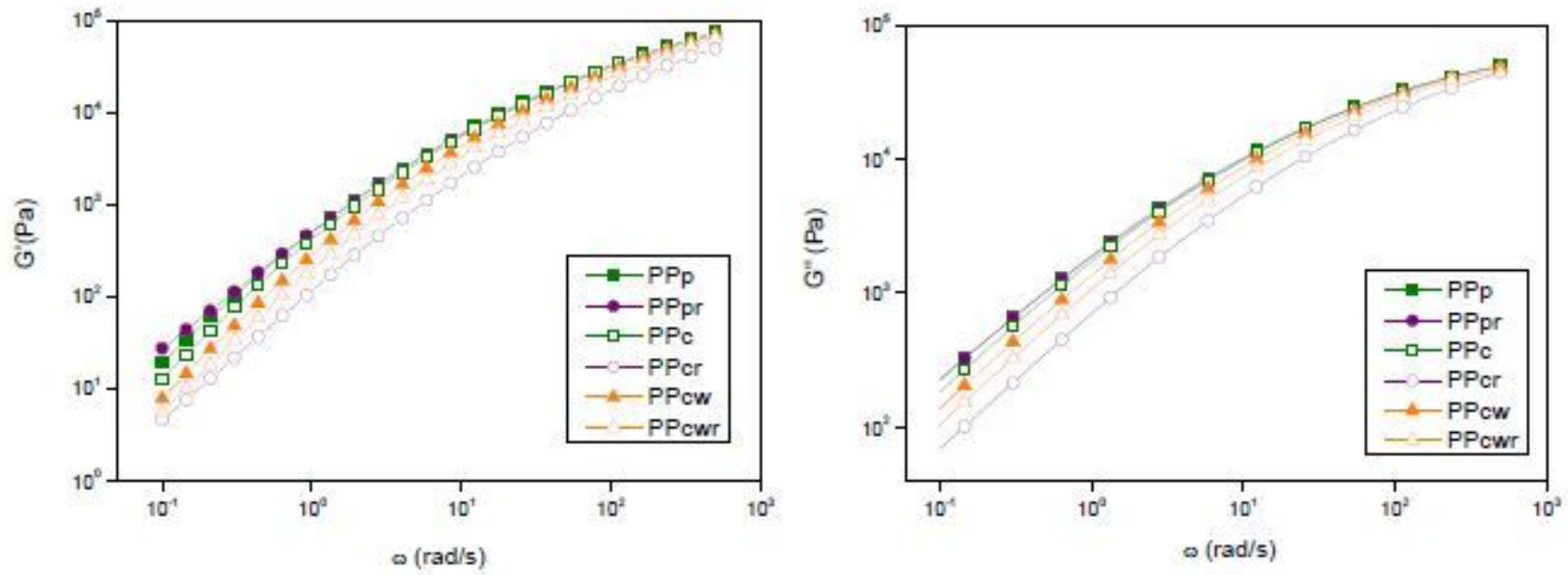

(a)

(b)

Figure 4

Storage modulus ( $\left.G^{\prime}\right)$ as a function of the frequency for all samples (a) and loss modulus ( $\left.G^{\prime \prime}\right)$ as a function of the frequency for all samples (b). 